\title{
Automated Building Footprint and 3D Building Model Generation from Lidar Point Cloud Data
}

\author{
Fayez Tarsha Kurdi* and Mohammad Awrangjeb \\ Institute of Integrated and Intelligent Systems \\ Griffith University, Nathan QLD 4111, Australia \\ \{f.tarshakurdi, m.awrangjeb\}@ griffith.edu.au
}

\author{
Alan Wee-Chung Liew \\ School of Information and Communication Technology \\ Griffith University, Southport, QLD 4215, Australia \\ a.liew@griffith.edu.au
}

\begin{abstract}
Although much effort has been spent in developing a stable algorithm for 3D building modelling from Lidar data, this topic still attracts a lot of attention in the literature. A key task of this problem is the automatic building roof segmentation. Due to the great diversity of building typology, and the noisiness and heterogeneity of point cloud data, the building roof segmentation result needs to be verified/rectified with some geometric constrains before it is used to generate the $3 \mathrm{D}$ building models. Otherwise, the generated building model may suffer from undesirable deformations. This paper suggests the generation of 3D building model from Lidar data in two steps. The first step is the automatic $2 \mathrm{D}$ building modelling and the second step is the automatic conversion of a $2 \mathrm{D}$ building model into 3D model. This approach allows the $2 \mathrm{D}$ building model to be refined before starting the $3 \mathrm{D}$ building model generation. Furthermore, this approach allows getting the 2D and 3D building models simultaneously. The first step of the proposed algorithm is the generation of the 2D building model. Then after enhancing and fitting the roof planes, the roof plane boundaries are converted into 3D by analysing the relationships between neighbouring planes. This is followed by the adjustment of the 3D roof vertices. Experiment indicated that the proposed algorithm is accurate and robust in generating 3D building models from Lidar data.
\end{abstract}

Keywords — Lidar, modelling, segmentation, 2D building model, 3 building model, vertex, boundary

\section{INTRODUCTION}

After segmenting the $3 \mathrm{D}$ point cloud data into three main classes, i.e., terrain, vegetation and buildings, a 3D model of the urban scene can be generated. This classification can go further by adding other classes for covering all city features such as powerlines, traffic lights, lighting columns and advertising billboards. This list can be expanded further to include more and more classes. Moreover, each one of the main classes can also be subdivided into several subclasses, e.g., the terrain class can be subdivided into terrain, roads, sidewalks, railways, bridges and footpaths. A new subdivision can then be achieved for each main class. In this way, an urban scene can be decomposed into a list of classes and subclasses that describe all its components.

Due to the significant variation of geometric characteristics and modelling concept of different urban scene classes, each class must have its own modelling method. Once each class and subclass in an urban scene is modelled, the regrouping of all these models allows the generation of the total city model.

In the literature, most research focuses on the modelling of buildings class, because this class covers most of the city area and is considered to be the main component of the city scene, representing the most important layer of the city model. Despite the occasional existence of very complex building architectural designs, where the building roofs contain sculptures, statues, domes, cones, cylinders, or mathematical curved surfaces instead of planer surfaces, the fundamental hypothesis in building modelling is that the building roof is composed of a set of planes. This hypothesis is based on the abundance of building roofs with shape formed by connected planes. The configuration of these planes define the roof geometry. Therefore, building modelling is concerned with the construction of a 3D building model consisting of planes, edge-lines, and vertices, starting from the point cloud data obtained from the building roof.

Two major approaches of building modelling can be found in the literature: the model-driven or parametric approach and the data-driven or non-parametric approach. The modeldriven approach processes the entire building point cloud and obtains a geometrical model without visual deformations by using a building library. The data-driven approach tries to fit each part of the building point cloud to get a faithful polyhedral model. However, it sometimes provides models with visual deformations and requires more running time. Nevertheless, data-driven approaches can process the majority of building forms whilst model-driven approaches process only the primitive building cases.

In data-driven approaches, the crucial procedure is the detection of roof planes, which drives the detection of the plane boundary lines and vertices. Success at this step is critical to the success of all the following processing steps. That is the reason Tarsha Kurdi et al. [16] suggested to validate the roof plane detection procedure through calculating firstly the 2D building model. Once a 2D building model is accurately calculated, the 3D building model can then be constructed.

This paper is focused on defining the pathway from 2D building modelling to 3D building modelling using Lidar data. This procedure depends on the analysis of the relationships between neighbouring roof planes (intersection, step and stepintersection). Then, the 3D building model can be constructed by using the enhanced roof plane equations.

\section{RELATED WORK}

In the context of 3D building modelling from Lidar data, model-driven and data-driven approaches are the two main approaches:

Model-driven approach: Several authors, e.g., Park et al. [7] and Kadaa and McKinley [4], suggested the segmentation of the complex building point cloud into primitives using ground planes or another type of additional data. Xiong et al. [17] suggested a model-driven approach based on finding the 
roof topology graphs. These graphs have three basic elements: the loose nodes, the loose edges, and the minimum cycles. A building was decomposed into a group of building parts predefined in a given library of building primitives. Then, constraints were applied to simultaneously adjust the whole roof. Zhang et al. [19] represented building roofs by using geometric primitives, then constructed a cost function by using constraints from both Lidar data and aerial imagery simultaneously. Zheng et al. [20] proposed a hybrid approach of data- and model-driven approaches. The input data of this algorithm were the Lidar data, the 2D building footprint and a high resolution orthophoto. The process extracted the step edge lines on the building roof from Lidar and 2D building footprints using the Canny filter. Then, three types of subfootprints were created, for each one a roof model selection procedure was performed to analyse the $3 \mathrm{D}$ roof primitives stored in the predefined library.

Data-driven approach: The approach developed by Ghaffarian et al. [2] utilised the Purposive Fast Independent Component Analysis algorithm (PFICA) for detecting roof plane boundaries, then a morphological filter was applied in order to enhance the detected boundaries. Finally, the Douglas-Peucker algorithm was used to localise their breakpoints. Xiao et al. [18] started also by extracting the roof planes. Then, plane boundaries and vertices were calculated by using an undirected graph model of the building roof. Consequently, the RANSAC algorithm was applied to obtain a reasonable and usable model with regular building boundary segments, and to find long segments and the corresponding parameters. Furthermore, another constrains were performed by applying parallel merging and orthogonal adjustments. Sampath and Shan [11] detected roof planes and plane boundaries simultaneously by using exclusively Lidar data. They applied the linear space theory to separate plane boundaries from planar points.

Some studies developed solutions for analysing topological relationships between building roof planes. Jaya et al. [3] and Perera et al. [8] used the roof topology graph based on the closed cycle graphs summarising the topological relationships between the roof segments. In the same context, the use of aerial images was suggested in addition to Lidar data. Schenk and Csatho [12] detected the roof plane boundaries from panchromatic images based on the texture discontinuity and utilised them to refine the results from Lidar data. Sohn and Dowman [13] employed the IKONOS multispectral images for roof plane boundary detection. Sohn et al. [14] and Li et al. [6] applied region growing and TIN analysis for extracting roof planes. While Sohn et al. [14] applied Hough transform and neighbouring plane intersection lines in order to extract planes boundaries, $\mathrm{Li}$ et al. [6] enhanced roof planes then the boundary was refined by iterative merging and updating the normals of boundary segments. Tarsha Kurdi et al. [16] preferred to calculate the $2 \mathrm{D}$ building model before converting it to $3 \mathrm{D}$ space.

\section{DATASETS}

In order to test our approach on different point clouds, two datasets are used (Table I). The first dataset Hermanni is a residential area in the periphery of Helsinki, where large and spaced storey houses are surrounded by vegetation. This point cloud belongs to the building extraction project of EuroSDR (www.eurosdr.org).

\begin{tabular}{|c|c|c|}
\hline & Hermanni & Vaihingen \\
\hline Acquisition & End of June 2002 & 21 August 2008 \\
\hline Sensor & TopoEye & $\begin{array}{c}\text { Leica Geosystems Leica } \\
\text { ALS50 system }\end{array}$ \\
\hline Points density & $7-9$ points $/ \mathrm{m}^{2}$ & $6.7-4$ point $/ \mathrm{m}^{2}$ \\
\hline Flight height & $200 \mathrm{~m}$ & $500 \mathrm{~m}$ \\
\hline
\end{tabular}

The second dataset was captured over Vaihingen city in Germany [1]. This area is characterised by a few residential buildings, historic buildings and small detached houses that are surrounded by trees.

\section{Motivations}

It is well known that the roof point cloud segmentation leads to the calculation of the 3D plane equation for each roof plane segment. Unfortunately, these plane equations do not always represent the roof segment surfaces accurately due to the following reasons:

- The planimetry and altimetry accuracy of Lidar point cloud.

- The presence of small roof details (as windows and chimneys) which are undetectable due to their small areas in comparison to the point density. Consequently, they are considered as noise.

- The roof texture sometimes has a considerable thickness value, which may be up to $15 \mathrm{~cm}$.

- The point density of vertical planes (as building façade planes, vertical walls surrounding roof details and the connection plane of two neighbouring planes of "step" mutual relationship) are low and its homogeneity level is very weak. Consequently, they are considered as noise.

- The vertical roof planes generate noise due to laser beam spot diameter.

- The heterogenic distribution of point cloud.

- The presence of noisy points due to random reasons.

- Regardless to all above reasons, the perfect mathematical plane surfaces can be rarely found anywhere. Therefore, the roof plane segments do not represent perfect mathematical plane surfaces.

Thus, if the roof neighbouring segment equations are used for calculating the 3D roof vertices coordinates $(X, Y$ and $Z)$, then there is a high probability to obtain a distorted result, and the $3 \mathrm{D}$ roof model will be deformed. In order to avoid this problem, the suggested algorithm proposes calculating the coordinates $(X$ and $Y$ ) of roof vertices from the building label image. The accuracy of the label image is tested and verified by the same algorithm, and the $Z$-value will be deduced from neighbouring segment equations.

Moreover, in the data-driven approach, the critical step is the detection of building roof planes, as any misdetection of the roof plane during this step will generate undesirable deformations in the 3D building model. Therefore, a decision 
is taken to carry out the building modelling into two consecutive steps: the first step is the 2D building modelling, which calculates the 2D building model. The second step is the conversion of the $2 \mathrm{D}$ building model into the $3 \mathrm{D}$ model. For this purpose, the mutual relationships of the neighbouring roof planes will be analysed, and modelled by using the refined fitted roof plane equations in addition to the calculated roof footprint.

The approach developed in this paper has several advantages. First, it controls and evaluates the roof segmentation result before calculating the 3D building model. Hence, if the segmentation result is not acceptable, it will be rejected and the 3D building model will not be calculated. Second, this algorithm helps to adjust the roof segmentation result and to refine the roof plane equations. Third, it provides a new output which is the $2 \mathrm{D}$ building model which can be added as a visualisation option to the 3D building model. Finally, it opens the door towards adding other geometric constrains during 2D building modelling, which helps to improve the quality of the 2D and 3D building models.

At this stage, the novelty of this paper can be summarised as the following:

- The 3D roof vertices coordinates are calculated in two steps: in the first one, the building label image is employed for calculating the ( $X$ and $Y$ ) coordinates, and in the next step, the $Z$-value is deduced from the roof neighbouring segment equations. This operation reduces remarkably the roof model deformations due to the distortion of roof segment equations.

- Controls and evaluates the roof segmentation result before calculating the 3D building model.

- The suggested algorithm assures the calculation of 3D building model just in case of successful roof segmentation.

\section{Automatic 2D BuILDING MODELLING}

The first step toward automatic generation of $2 \mathrm{D}$ building model is the automatic detection of the roof planes. In order to do this, the extended RANSAC algorithm is used [15]. This algorithm allows the harmonisation of the mathematical aspect of the RANSAC algorithm with the geometry of a roof. The input data of this algorithm is the $3 \mathrm{D}$ building Lidar point cloud, its output is the building label image shown in Fig. 1a.

Once the building point cloud is segmented, the next step is the calculation of a 2D building model. To achieve this, the adjacency relationships between roof planes have to be examined by using a $3 \times 3$ moving window. This step allows the neighbouring planes to be determined by calculating the
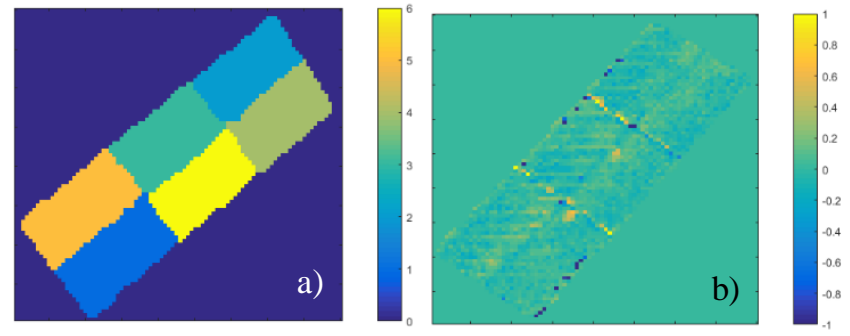

Fig. 1. Segmentation of the building point cloud. a) Building label image (the colors present the plane numbers). b) Visualisation of error map (the colors present the deviation values) neighbourhood matrix. This matrix is a binary matrix, that contains only two values ( 0 and 1$)$. The number of columns or lines of this matrix is equal to the number of roof planes. For example, if two planes 1 and 2 are adjacent, then the cell (1, 2) of the neighbourhood matrix is equal to 1 .

Then, the inner roof plane boundaries are detected. Finally, the junction relationships between roof plane boundaries are analysed in order to detect the roof vertices. For more details about this step, see Tarsha Kurdi et al. [16].

In order to model the building outer boundaries, firstly the building boundary polygon is detected and filtered. Then, the Douglas Peucker technique [23] is employed in order to calculate the coordinates of the extremity points as well as the list of all points describing every outer boundary segment line. After that the least squares theory is applied to calculate the mean line of each segment. Then, the building outer boundary is enhanced by merging the boundary segments that belong to the same façade plane. For more details about this step, see Tarsha Kurdi et al. [22]. Figs. 2b and 3a show the calculated 2D building model starting from the building label image presented in Fig. 2a.

After constructing the 2D building model, it is necessary to estimate the segmentation accuracy; hence, the error map matrix is calculated. This matrix allows a pixel by pixel analysis of the deformations and the accuracy of the building roof segmentation.

In order to calculate the error map matrix, the distance (deviation) between each point and its mean plane is calculated and assigned to the corresponding pixel. To visualise the error map, the variances are clipped to be between $-1 \mathrm{~m}$ to $+1 \mathrm{~m}$ (see Fig. 1b). This matrix provides a visual and numerical estimation of the accuracy of building roof segmentation, and facilitates the adjustment and refinement of the building roof segmentation and the roof plane equations as described in Section VI below.

In summary, at the end of this operation, four classes will be defined: the outer building boundaries, the roof planes, the $2 \mathrm{D}$ inner roof plane boundaries and the $2 \mathrm{D}$ roof vertices. Once the $2 \mathrm{D}$ roof model is calculated, the next step is the adjustment of building roof segmentation before calculating the $3 \mathrm{D}$ building model.

\section{ADJUSTMENT OF BUILDING ROOF SEGMENTATION}

Before converting the $2 \mathrm{D}$ building model into $3 \mathrm{D}$ one, it is necessary to achieve a set of controls and tests on the $2 \mathrm{D}$ roof segmentation result. In this context, the authors in Refs. [5, 6, 10] suggested the use of a graph cut approach to optimise the delineation of roof elements initialised on a 2D supporting grid. However, the suggested approach in this paper differs from the existing papers $[5,6,10]$, as it does not limit the optimisation of the roof segmentation result in $2 \mathrm{D}$, but it goes very far by constructing, evaluating and optimising the $2 \mathrm{D}$ building model. In the context of control and test of the $2 \mathrm{D}$ roof segmentation result, an analysis of pixel values in the error map shows that the majority of values are between $(-0.25$ $\mathrm{m})$ and $(+0.25 \mathrm{~m})$. This interval is related to the altimetry accuracy of the point cloud. Hence, the pixel values in the error map can be classified into two classes:

- Pixels having values in the interval $[-0.25,+0.25]$ represent the majority of error map values. 
- Pixels having values outside the interval [- 0.25, + $0.25]$ represent noise.

It is noted that the points having deviations outside the interval $[-0.25,+0.25]$ generate distortion in the plane equations. These points can be excluded after the first plane fitting, then the plane equation is refitted again. However, this solution sometimes creates gaps among the plane area and as a consequence the distribution of points among the plane area will not be balanced. Moreover, the number of excluded points can sometimes be large. We therefore propose a new solution to solve this problem. In order to adjust the building roof segmentation, every point having noise value outside the interval $[-0.25,+0.25]$ have to be projected onto its corresponding plane. This operation will allow us to generate a new adjusted and balanced building point cloud. We can then re-segment the new point cloud of the building.

Let us take the example presented in Fig. 2a, which represents the visualisation of the re-segmentation of building point cloud presented in Fig.1. Fig. 2b presents the adjusted 2D building model. Here, the total number of building points is equal to 2674 points and the number of adjusted points is equal to 84 points. Table II compares the two 2D building models before and after the adjustment. It can be observed that the number of points of each principal plane is different before and after the adjustment because the plane equations are enhanced. It can also be noted that the standard deviation values of planes fitting become smaller. Furthermore, this adjustment will improve the quality of the 2D building model. This conclusion can be noted clearly by comparing the adjusted building label image in Fig. 2a with the building label image before adjustment in Fig. 1a.

At this stage it is important to underline the main idea of this approach which is to perform the building modelling in two steps: automatic 2D building modelling and, then, automatic 3D building modelling. The adjustment of 2D building model represents a link task between the two last steps.

In this paper, it is suggested only to enhance the roof segmentation and by consequence refining the roof plane equations. But other controls, enhancements and geometric constrains can be added to the adjustment task for avoiding whole foreseeable deformation in the level of 3D building model.

Once the 2D building roof segmentation is adjusted and the $2 \mathrm{D}$ building model is calculated, the construction of $3 \mathrm{D}$ building model can be started.
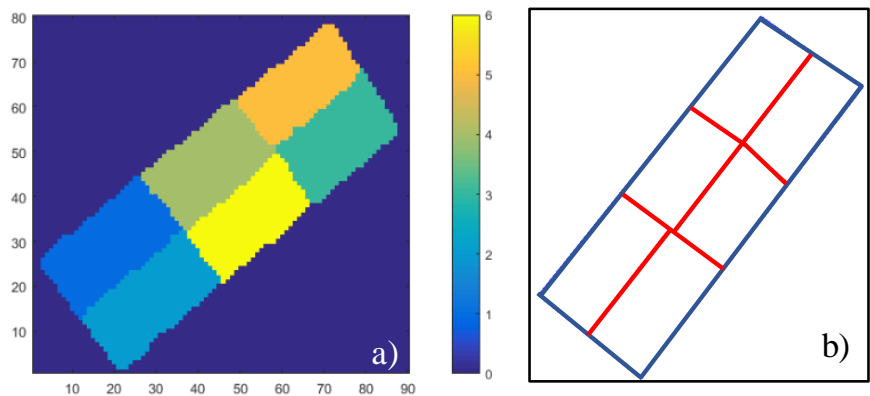

Fig. 2. Adjustment of segmentation of the building point cloud. a) Adjusted building label image (the colors present the plane numbers). b) 2D building model, (blue lines presents outer building boundary and red lines present inner roof boundaries)

\begin{tabular}{|c|c|c|c|c|}
\hline & \multicolumn{2}{|c|}{$\begin{array}{c}\text { Before } \\
\text { adjustment }\end{array}$} & \multicolumn{2}{c|}{ After adjustment } \\
\hline $\begin{array}{c}\text { Plane } \\
\text { number }\end{array}$ & $\begin{array}{c}\text { Number } \\
\text { of points }\end{array}$ & $\begin{array}{c}\text { S. } \boldsymbol{d} \\
(\boldsymbol{m})\end{array}$ & $\begin{array}{c}\text { Number } \\
\text { of points }\end{array}$ & $\begin{array}{c}\text { S. } \boldsymbol{d} \\
(\boldsymbol{m})\end{array}$ \\
\hline 1 & 508 & 0.66 & 513 & 0.14 \\
\hline 2 & 488 & 0.10 & 483 & 0.09 \\
\hline 3 & 429 & 0.13 & 426 & 0.08 \\
\hline 4 & 406 & 0.49 & 409 & 0.17 \\
\hline 5 & 416 & 0.12 & 441 & 0.09 \\
\hline 6 & 427 & 0.37 & 402 & 0.16 \\
\hline
\end{tabular}

Where $\boldsymbol{S} . \boldsymbol{d}$ is the standard deviation

\section{AUTOMATIC 3D BUILDING MODELLING}

As it is shown in the last section, the construction of the 2D building model allows us to define four entity classes corresponding to the $2 \mathrm{D}$ building model components: the outer building boundaries, the roof planes, the inner roof plane boundaries and the roof vertices. This section describes how the 3D building model can be obtained starting from the 2D building model constructed previously. This is done in three steps. Firstly, the mean adjusted roof plane equations have to be fitted. The second step is the calculation of the roof plane boundaries and the roof vertices in the 3D space. Finally, the $3 \mathrm{D}$ roof vertices have to be adjusted. The following three subsections detail these steps.

\section{A. Calculation of the roof plane equations}

The extended RANSAC algorithm which was used for detecting the roof planes does not yield the mean roof plane equations (best fitting planes) but it delivers sets of roof plane points in addition to the supplementary information which is used for identifying the principal roof planes from the detail ones. For this reason, it is necessary to calculate firstly the equations of the mean principal roof planes by using the least square theory. This calculation is carried out in two iterations. After the first one, the noisy points are eliminated.

Concerning the roof detail planes (such as chimneys and windows), all planes of this family are considered to be horizontal (parallel to the $O X Y$ plane). Then, the detail plane altitudes are taken as the mean altitude of the detail point cloud. This decision has been adopted with the following reasons: First, the areas of roof details are small, taking into account the point density, then the number of points representing the roof detail plane is very small in comparison to the principal roof plane. Second, the geometric form of roof details are vastly varying. Finally, the scanned points of vertical walls surrounding roof detail sometimes generate considerable noise. All these reasons motivated us to suppose that all roof details planes are horizontal. Once all roof plane equations are fitted, the 3D relationships between neighbouring roof planes can be analysed.

\section{B. Conversion of roof plane boundaries to $3 D$}

As each plane boundary line in 2D plane separates two neighbouring roof planes, we assume that each plane boundary line in $2 \mathrm{D}$ space $(O X Y)$ represents two lines in the $3 \mathrm{D}$ space $(O X Y Z)$ (see Fig. 3). The $Z$ value of each $2 \mathrm{D}$ boundary line extremity is calculated twice from each one of 
two neighbouring plane equations. The analysis of the position of these two 3D lines will define the relationship between the two neighbouring planes. According to Rottensteiner [9], three types of mutual relationships can be defined: intersection, step, and step-intersection (see Fig. 4). Equation (1) permits us to determine the type of mutual relationships between two adjacent planes, where $Z_{i j}$ is mentioned in Fig3.

$$
\Delta Z_{1}=Z_{11}-Z_{21} \text { and } \Delta Z_{2}=Z_{12}-Z_{22}
$$

The value of $\Delta Z_{i}$ allows us to adjust the two 3D boundary lines as shown in Fig. 5 according to the following five cases:

- Case a: If $\Delta Z_{1} \times \Delta Z_{2}<0$ and the intersection point is far from the midpoint of the four extremities, then the closest extremities are merged by taking the mean of their altitudes.

- Case b: If $\left(\left|\Delta Z_{1}\right|<T_{h},\left|\Delta Z_{2}\right| \geq T_{h}\right.$ and $\Delta Z_{1} \times \Delta Z_{2}>$ 0 ) or $\left(\left|\Delta Z_{1}\right| \geq T_{h},\left|\Delta Z_{2}\right|<T_{h}\right.$ and $\left.\Delta Z_{1} \times \Delta Z_{2}>0\right)$, then the closest extremities are merged by taking the mean of their altitudes.

Cases (a) and (b) represent the "step-intersection" relationship.

- Case c: If $\Delta Z_{1} \times \Delta Z_{2}<0$ and the intersection point is close to the midpoint, then the extremities are merged as $Z_{1}=\operatorname{mean}\left(Z_{11} ; Z_{21}\right)$ and $Z_{2}=\operatorname{mean}\left(Z_{12} ; Z_{22}\right)$.

- Case d: If $\left|\Delta Z_{1}\right|<T_{h},\left|\Delta Z_{2}\right|<T_{h}$ and $\Delta Z_{1} \times \Delta Z_{2}>$ 0 , then the extremities are merged as $Z_{1}=$ $\operatorname{mean}\left(Z_{11} ; Z_{21}\right)$ and $Z_{2}=\operatorname{mean}\left(Z_{12} ; Z_{22}\right)$.

Cases (c) and (d) represent the "intersection" relationship.

- Case e: If $\left|\Delta Z_{1}\right| \geq T_{h},\left|\Delta Z_{2}\right| \geq T_{h}$ and $\Delta Z_{1} \times \Delta Z_{2}>$ 0 , then no adjustment will be carried out.

Case (e) represents the "step" relationship.

Where $T_{h}$ is a given threshold related to the altimetry accuracy. Its value in our example is equal to $40 \mathrm{~cm}$.

In summary, three operations have been carried out in this step: calculation of the inner plane boundaries in $3 \mathrm{D}$, analysis of the mutual relationship between neighbouring roof planes and adjustment of 3D plane boundaries according to their mutual relationships. In the next step, the boundary 3D line extremities located on the same vertex will be adjusted.
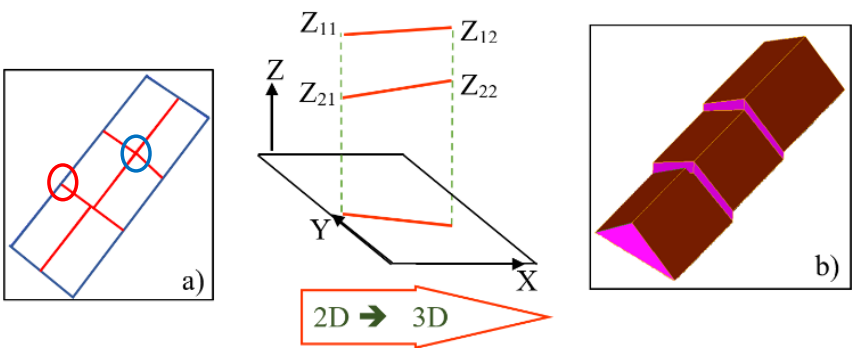

b)

Fig. 3. Conversion of the roof plane boundaries and vertices from $2 \mathrm{D}$ to $3 \mathrm{D}$; a) $2 \mathrm{D}$ roof model; b) $3 \mathrm{D}$ roof model

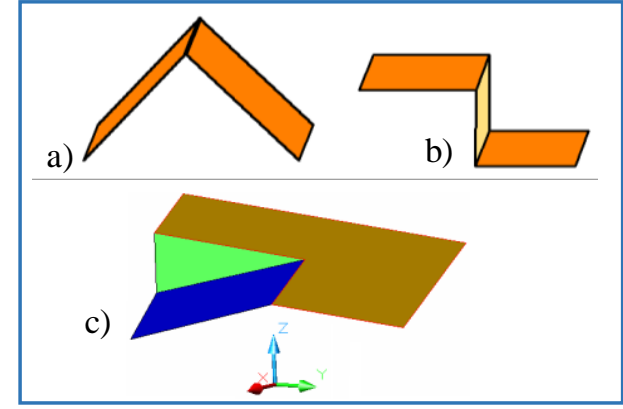

Fig. 4. Mutual relationship of two neighbouring roof planes. a) Intersection; b) Step; c) Step-intersection (a and b are from [15])

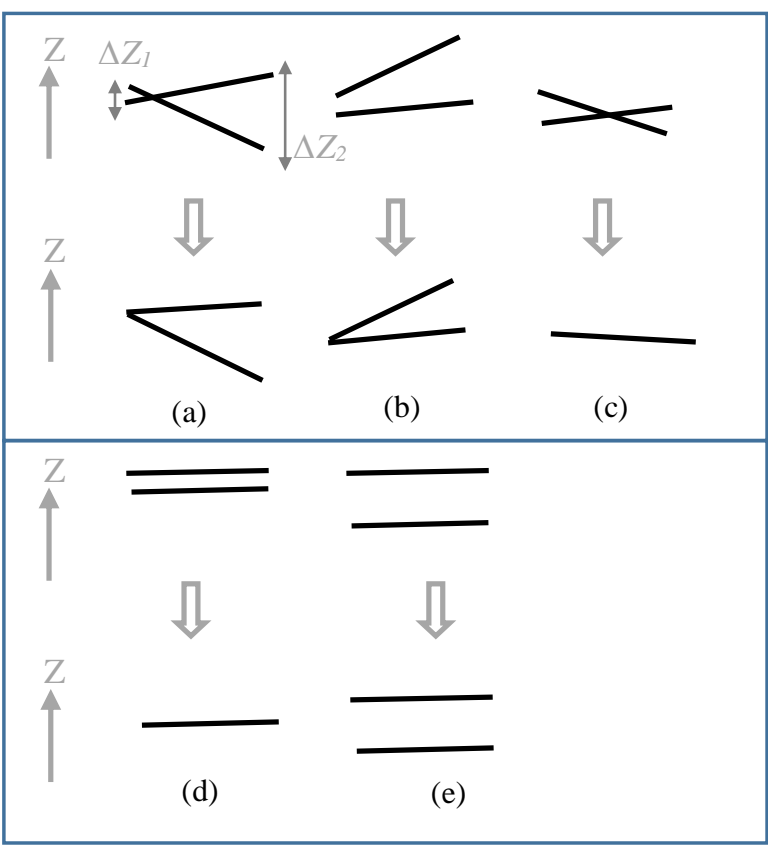

Fig. 5. Adjustment of the 3D boundary lines according to the relationship: a) and b) Step-intersection; c) and d) Intersection; e) Step

\section{Adjustment of $3 D$ roof vertices}

The roof vertices can be classified into two classes: inner vertices which are located inside the roof boundary, e.g., inside the blue circle in Fig. 3a, and outer vertices which are located on the building boundary, e.g., inside the red circle in Fig. 3a. Furthermore, in Fig. 5 it can be noticed that the extremities of 3D boundary lines can be classified into two classes: the first class is the extremity that will be merged with the other one, it is called $m_{e x t}$, e.g., the two extremities of Cases $\mathrm{c}$ and $\mathrm{d}$, and the left extremities of Cases $\mathrm{a}$ and $\mathrm{b}$ in Fig. 5. The second class is the extremity that will not be merged with the other one, it is called $n_{\text {ext }}$, e.g., the two extremities of Case e and the right extremities of Cases $a$ and $b$ in Fig. 5.

Since one vertex represents the intersection of $n$ neighbouring planes in the $2 \mathrm{D}$ building footprint, where $n \geq$ 2 , it therefore represents the intersection of $n$ boundary lines in the 2D building footprint. As the extremity of each inner boundary 2D line will be calculated twice in 3D (see Fig. 3), the coordinates of each vertex will be calculated $2 n$ times in $3 \mathrm{D}$, and all the generated $3 \mathrm{D}$ points have the same planer coordinates $(X$ and $Y$ ).

In order to adjust the lines extremities of the 3D inner vertices, three cases are considered: 
- Case 1: All line extremities are of class $m_{\text {ext }}$, e.g., inside the blue circle in Fig. 9b, all line extremities will be merged into one point in the $3 \mathrm{D}$ space.

- Case 2: All line extremities are of class $n_{\text {ext }}$, e.g., the roof details extremities in Fig. $7 \mathrm{a}$ are of class $n_{\text {ext }}$. In this case, all line extremities will be conserved without merging.

- Case3: The line extremities are a mixture of $n_{\text {ext }}$ and $m_{\text {ext }}$, e.g., inside the blue circle in Fig. 3a, the merging operation has to be done in two consecutive steps. In the first step, the two extremities of the two boundary lines surrounding each roof plane have to be merged as in Fig. 6c. In the second step, the pairs of extremities of class $m_{\text {ext }}$ have to be merged as in Fig. 6d.

Remark: in the case of "step" relationship, the vertex in the $2 \mathrm{D}$ building footprint will generate a vertical line linking the two lines extremities in 3D (the green line in Fig. 6e).

Fig. 7 shows the position of the 3D vertices (inside red circles) before and after adjustment. Once the inner roof vertices is checked and adjusted, the coordinates of the inner roof vertices may be changed, therefore the conditional least squares fitting is applied in order to update the roof plane equations by respecting the location of vertices related to Cases 1 and 3 shown above. Then, the vertices coordinates of Case 2 are updated.

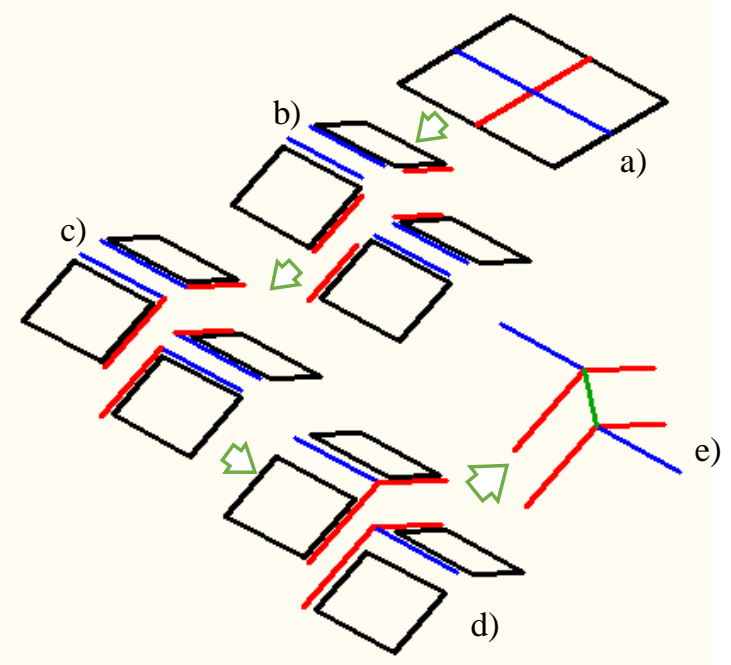

Fig. 6. Two extrimites of classes $n_{e x t}$ and $m_{\text {ext }}$ are presented in the same vertex. a) $2 \mathrm{D}$ roof model; b) multiplication of plane boundary lines in $3 \mathrm{D}$ space; c) Extremities merging of two boundary lines surrounding each roof plane; d) Merging the extremities of class $m_{\text {ext }}$; e) Final 3D model of the vertex

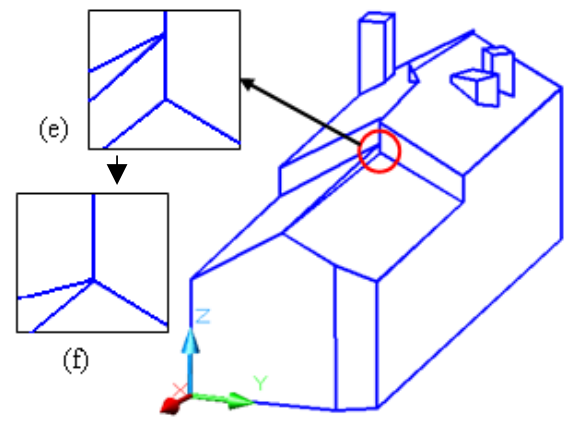

(a)

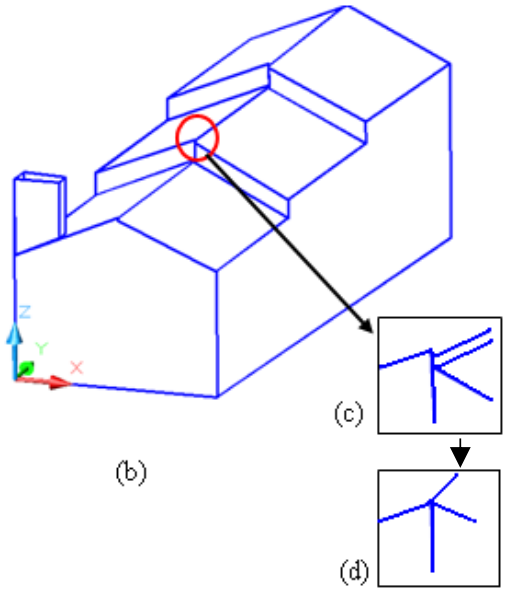

Fig. 7. Adjustment of roof vertices. a) and b) Building 6 and 3 in Fig. 8; c) and e) 3D vertices before adjustment; f) and d) 3D vertices after adjustment

For the outer vertices class, one vertex will be considered as breakpoint in the building outer boundary. Moreover, each outer building boundary line belongs only to one roof plane, and it will generate only one line in the $3 \mathrm{D}$ space. These two remarks, in addition to the three cases of inner vertices which are discussed above, are applied to construct the 3D building model.

Once the 3D building model is constructed, it is inevitable to analyse the modelling accuracy as a quality control procedure.

\section{RESULTS}

Fig. 8 illustrates the 3D models of the Hermanni site.

Figs. 9 and 10 illustrate the 3D building models of the Vaihingen site. Fig. $9 \mathrm{~b}$ shows the 2D and 3D building models together.

It can be noted from this result that the proposed algorithm allows constructing not only the principal roof planes but also some roof details depending on their dimensions and the point cloud density.
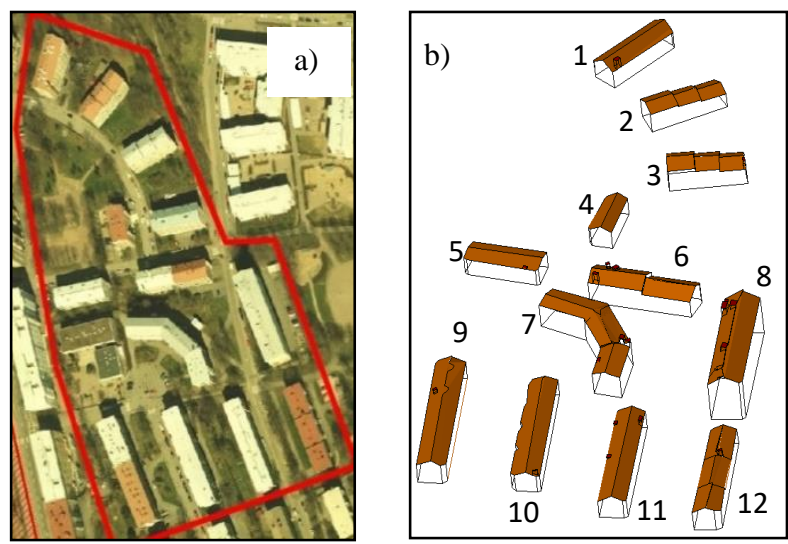

Fig. 8. 3D building models; a) Aerial image of Hermanni site; b) Total 3D building model 

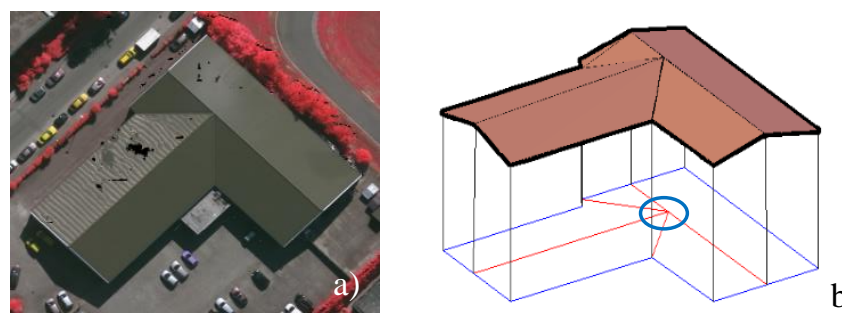

b)

Fig. 9. 3D building model of Building 1 of Vaihingen site; a) Aerial image; b) $2 \mathrm{D}$ and $3 \mathrm{D}$ building models
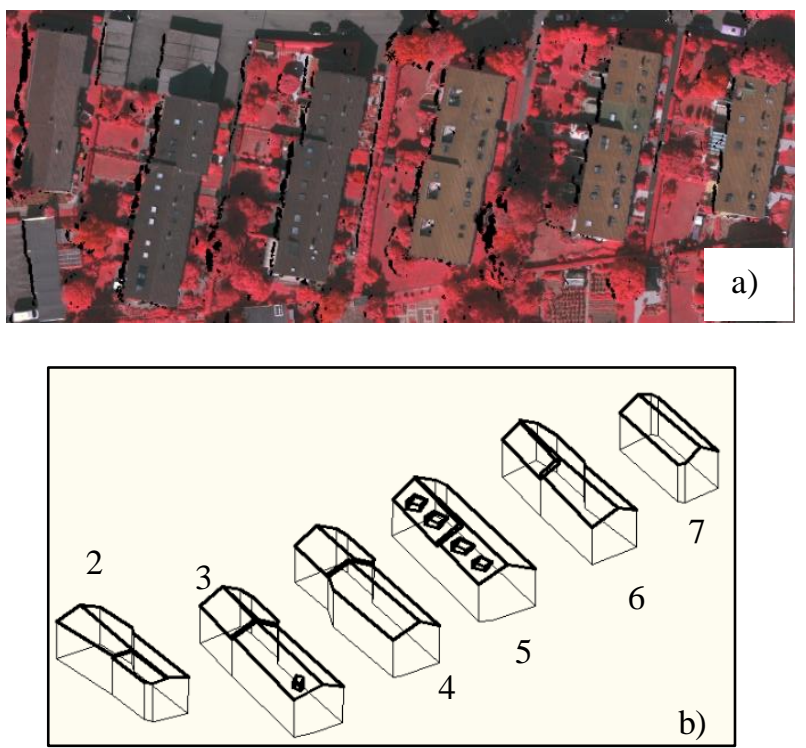

Fig. 10. 3D building models of buildings of the Vaihingen site; a) Aerial image; b) Total 3D building models

\section{ACCURACY AND ANALYSIS}

Ostrowski et al. [24] summaries the approaches of 3D building modelling accuracy estimation into two classes. In the first class, a reference model is needed in order to compare the calculated model with it. Whereas the approaches in the second class consider the Lidar point cloud as a reference data. Hence, the accuracy estimation approach employed in this section joints the second class where the building point cloud is considered as reference data.

In this context, in order to estimate the accuracy of the 2D building models, the error map is calculated for each building, then the percentage of pixels having considerable noise (deviation values outside the interval $[-0.25,+0.25])$ is calculated. This percentage allows us to evaluate the accuracy of roof segmentation before adjustment.

From Table III, it can be noted that more than $90 \%$ of building points (Buildings 6 and 3) have an acceptable deviation regarding the accuracy of the point positions. The application of the same test for all tested buildings also shows the same result. This result indicates the high quality of the obtained building models.

On the other hand, to estimate the accuracy of the 3D building models presented in Figs. 8, 9 and 10, the standard deviation of the $3 \mathrm{D}$ building model is calculated. Fig. 11 illustrates the standard deviation of the 3D building models of the Hermanni and Vaihingen datasets.

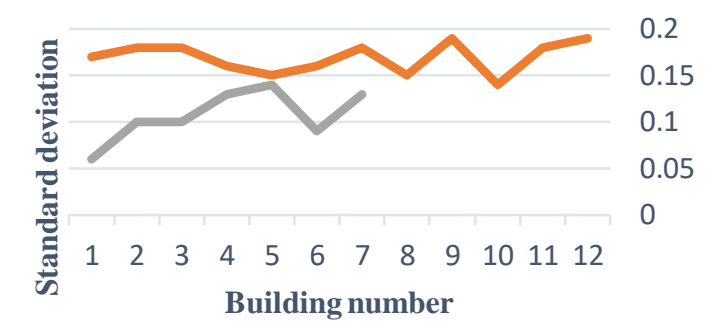

$\longrightarrow$ Vaihingen Hermanni

Fig. 11. Standard deviations of 3D building models

TABLE III. ANALYSIS OF ERROR MAP (DEVIATION DISTRIBUTION)

\begin{tabular}{|c|c|c|c|c|}
\hline \multirow{2}{*}{$\begin{array}{l}\text { Building } \\
\text { Number }\end{array}$} & \multicolumn{3}{|c|}{ Deviation values $(m)$} & \\
\hline & -1 To -0.25 & -0.25 To 0.25 & 0.25 To 1 & \\
\hline 6 & 4 & 90 & 6 & Point percentage \\
\hline 3 & 2 & 95 & 3 & \\
\hline
\end{tabular}

It shows that the models of the Vaihingen site are more accurate than the Hermanni site despite that the flight height of the Hermanni data $(200 \mathrm{~m})$ is smaller than that of the Vaihingen data $(500 \mathrm{~m})$. The reason for this accuracy difference comes from the quality of the Lidar sensor (see Table I). The modelling accuracy of the datasets has a standard deviation smaller than $0.2 \mathrm{~m}$ which can be considered as excellent given the point cloud accuracy of $40 \mathrm{~cm}$.

Regarding roof details modelling, e.g., chimneys, windows and antennas, Figs. 8 and 10 show that only some of the roof details are modelled. Indeed, according to the point cloud density and the roof detail dimensions, we can distinguish between two roof detail types: detectable roof details and undetectable ones. The roof segmentation algorithm can distinguish the roof details if they have areas greater than the given threshold in addition to having a considerable altitudes.

Unfortunately, if the roof details are not detected, they will be considered as undesirable noise and will affect the accuracy of the 3D building model. Furthermore, the presence of undetectable roof details in the point cloud data generates deformations in the $2 \mathrm{D}$ as well as in the $3 \mathrm{D}$ building model. As a consequence, the deformations encountered here would appear sometimes as reflection of presence of the indiscernible details on the building roof, whereas they do not appear always as geometric uncertainty as it is cited by Rottensteiner et al. [21].

\section{CONCLUSION AND PERSPECTIVE}

We presented in this paper a new approach for the generation of $2 \mathrm{D}$ and $3 \mathrm{D}$ building model from Lidar data. As the proposed approach belongs to the data-driven approach, it does not need a library of predefined building models. The proposed approach performs 3D building modelling in two consecutive steps: 2D building modelling and, then, 3D building modelling. This approach leads to several advantages, i.e., evaluation of the roof segmentation result before constructing the 3D building model, adjustment of the 
roof segmentation result and refinement of the roof plane equations, and finally the possibility of adding additional geometric constrains during $2 \mathrm{D}$ building model generation. All these benefits improve the quality of the $2 \mathrm{D}$ as well as the 3D building models.

The accuracy analysis has shown that the results are very satisfactory and reliable. It has also shown that even without any additional data, the proposed algorithm is able to generate automatically highly accurate 3D building models from Lidar data. Nevertheless, the proposed algorithm can only model the detectable roof details, and the undetectable roof details are treated as undesirable noise, which could degrade the accuracy of the generated 3D building models. A possible way to deal with this problem is to model them using roof details chosen from a predefined model library. Another future work is to integrate additional geometric constraints into the modelling pipeline to further improve the $2 \mathrm{D}$ and the $3 \mathrm{D}$ building modelling processes and make them more faithful to the reality. Afterwards, the research project will focus on the extraction of other natural and artificial objects in the city, in particular vegetation, powerlines and roads.

\section{ACKNOWLEDGMENT}

The Vaihingen data set was provided by the German Society for Photogrammetry, Remote Sensing and Geoinformation (DGPF) [1]. http://www.ifp.unistuttgart.de/dgpf/DKEP-Allg.html.

\section{REFERENCES}

[1] M. Cramer, "The DGPF test on digital aerial camera evaluation overview and test design," Photogrammetrie - Fernerkundung Geoinformation 2(2010):73-82.

[2] S. Ghaffarian, S. Ghaffarian, Y. Elmerabet, Z. Samir and Y. Ruicheck, "Automatic building roof segmentation based on PFICA algorithm and morphological filtering from Lidar point clouds," in 37th Asian Conference on Remote Sensing (ACRS 2016), promoting spatial data infrastructure for sustainable economic development, 17-21 October 2016, Colombo, Sri Lanka.

[3] S.N. Jaya, J. Akshay and K. Beena, "Contour Extraction in Buildings in Airborne Lidar Point Clouds Using Multiscale Local Geometric Descriptors and Visual Analytics," IEEE Journal of Selected Topics in Applied Earth Observations and Remote Sensing, PP. 1-16. 10.1109/JSTARS.2018.2833801.

[4] M. Kadaa and L. McKinley, "3D building reconstruction from Lidar based on a cell decomposition approach," in IAPRS, Vol. XXXVIII, Part 3/W4, Paris, France, 3-4 September, 2009.

[5] F. Lafarge and C. Mallet, "Creating large-scale city models from 3D point clouds: a robust approach with hybrid representation," in Int. J. Comput. Vision 99 (1), 69-85, 2012.

[6] M. Li, F. Rottensteiner and C. Heipke, "Modelling of buildings from aerial Lidar point clouds using TINs and label maps," in ISPRS Journal of Photogrammetry and Remote Sensing 154 (2019) 127-138. https://doi.org/10.1016/j.isprsjprs.2019.06.003.

[7] J. Park, I. Lee, Y. Choi and Y.J. Lee, "Automatic extraction of large complex buildings using Lidar data and digital maps," in Workshop ISPRS. Com III, Photogrammetric Computer Vision PCV Bonn, Germany 20 - 22 September 2006.

[8] S.N. Perera, H.A. Nalani and H.G. Maas, "An automated method for 3D roof outline generation and regularization in airborne laser scanner data," in Int. Arch. of the Photogrammetry, Remote Sensing, ISPRS Ann. Photogrammetry. Remote Sens. Spatial Inform. Sci. I-3, 281-286, 2012.

[9] F. Rottensteiner, "Automatic generation of high-quality building models from Lidar data," IEEE Computer Graphics and Applications, Vol. 23(6):42-51, 2003

[10] J. Shan and C.K. Toth, Topographic laser ranging and scanning principles and processing, second edition, by Taylor \& Francis Group, LLC. ISBN- 13: 978-1-4987-7227-3 (hardcover), 630 P, 2018.
[11] A. Sampath and J. Shan, "Clustering based planar roof extraction from Lidar data," in American Society for Photogrammetry and Remote Sensing, Annual Conference, Reno, Nevada, May 1-6-2006.

[12] T. Schenk and B. Csatho, "Fusion of Lidar data and aerial imagery for a more complete surface description," Int. Arch.of the Photogrammetry, Remote Sensing and Spatial Information Sciences, Proceedings of the ISPRS Commission III Symposium on Photogrammetric and Computer Vision, Volume XXXIV, Graz, Austria, 2002, pp. 310-317.

[13] G. Sohn and I. Dowman, "Data fusion of high-resolution satellite imagery and Lidar data for automatic building extraction," ISPRS Journal of Photogrammetry and Remote Sensing, 62(1), May, 43-63, 2007.

[14] G. Sohn, X. Huang and V. Tao, "Using a binary space partitioning tree for reconstructing polyhedral building models from airborne Lidar data," Photogrammetric Engineering and Remote Sensing, 1436 November 2008.

[15] F. Tarsha Kurdi, T. Landes and P. Grussenmeyer, "Extended RANSAC algorithm for automatic detection of building roof planes from Lidar data," The Photogrammetric Journal of Finland, vol. 21, n¹, 2008, pp. 97-109.

[16] F. Tarsha Kurdi, M. Awrangjeb and N. Munir, "Automatic 2D modelling of inner roof planes boundaries starting from Lidar data," 14th 3D GeoInfo 2019, 26-27 September, Singapore, in press.

[17] B. Xiong, M. Jancosek, S. Oude Elberink and G. Vosselman, "Flexible building primitives for 3D building modelling," ISPRS Journal of Photogrammetry and Remote Sensing 101 (2015) 275-290.

[18] Y. Xiao, C. Wang, J. Li, W. Zhang, X. Xi, C. Wang and P. Dong, "Building segmentation and modelling from airborne Lidar data," in International Journal of Digital Earth, 8:9, 694-709, DOI: 10.1080/17538947.2014.914252, 2015.

[19] W. Zhang, H. Wang, Y. Chen, K. Yan and M. Chen, "3D building roof modeling by optimizing primitive's parameters using constraints from Lidar data and aerial imagery," in Remote Sens. 2014, 6, 8107-8133; doi: $10.3390 /$ rs6098107.

[20] Y. Zheng, Q. Weng and Y. Zheng, "A hybrid approach for threedimensional building reconstruction in indianapolis from Lidar data," in Remote Sens. 2017, 9, 310; doi: 10.3390/rs9040310.

[21] F. Rottensteiner, J. Trinder, S. Clode, and K. Kubik,"Automated delineation of roof planes from lidar data," in Int. Arch. of Photogrammetry and Remote Sensing, vol. XXXVI, part 3/W19, pp. 221-226, 2005.

[22] F. Tarsha Kurdi, T. Landes and P. Grussenmeyer, "Joint combination of point cloud and DSM for 3D building reconstruction using airborne laser scanner data," in 4th IEEE GRSS / WG III/2+5, VIII/1, VII/4 Joint Workshop on Remote Sensing \& Data Fusion over Urban Areas and 6th International Symposium on Remote Sensing of Urban Areas. 1113 April, 2007 Télécom Paris. 1-4244-0712-5/07/\$20.00 @2007 IEEE, 7 pp.

[23] D.H. Douglas and T.K. Peucker, "Algorithms for the reduction of the number of points required to represent a digitized line or its caricature," in the Canadian Cartographer 10 (2), 112 -122. 1973.

[24] W. Ostrowski, M. Pilarska, J. Charyton and K. Bakuła, "Analysis of 3D building models accuracy based on the airborne Laser scanning point clouds," in the International Archives of the Photogrammetry, Remote Sensing and Spatial Information Sciences, Volume XLII-2, 2018, ISPRS TC II Mid-term Symposium "Towards Photogrammetry 2020", 4-7 June 2018, Riva del Garda, Italy. https://doi.org/10.5194/isprs-archives-XLII-2-797-2018. 